Review

\title{
The Roles of Inflammation, Oxidative Stress and the Gut-Brain Axis in Treatment Refractory Depression in Youth: Complementary and Integrative Medicine Interventions
}

\author{
Deborah R. Simkin ${ }^{1,}{ }^{*}$, L. Eugene Arnold ${ }^{2}$
}

1. Department of Psychiatry, Emory School of Medicine, 4641 Gulfstarr Dr. Suite 106, Destin, Florida 32541, USA; E-Mail: Deb62288@aol.com

2. Professor Emeritus of Psychiatry \& Behavioral Health, College of Medicine, Ohio State University, USA; E-Mail: L.Arnold@osumc.edu

* Correspondence: Deborah R. Simkin; E-Mail: Deb62288@aol.com

Academic Editor: Bettina Bernstein

Special Issue: Integrative Therapies to Address Mood and Anxiety Symptoms in Youth and Young People

OBM Integrative and Complementary Medicine 2020, volume 5 , issue 4

doi:10.21926/obm.icm.2004040
Received: April 06, 2020

Accepted: September 22, 2020

Published: October 19, 2020

\section{Abstract}

Teen depression and suicide rates have risen despite conventional treatments. This article reviews adjunctive interventions that may improve outcomes. A search of the National Library of Medicine database used tailored searches with combinations of specific terms. Modern lifestyle is associated with increased inflammation and pro-inflammatory cytokines leading to, for instance, hyperactivation of the hypothalamic-pituitary-adrenal axis, which promotes depression. Inflammation also increases oxidative stress, leading to mitochondrial dysfunction, also associated with depression. Diets with less probiotic-containing fermented foods change the microbiome and decrease the bio-availability of mood-regulating $B$ vitamins crucial to neurotransmitter production. Vitamin D deficiency allows increased pro-inflammatory cytokines

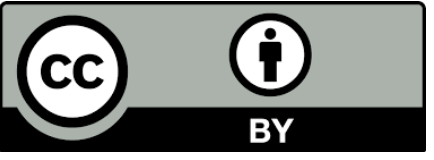

(C) 2020 by the author. This is an open access article distributed under the conditions of the Creative Commons by Attribution License, which permits unrestricted use, distribution, and reproduction in any medium or format, provided the original work is correctly cited. 
and disrupts mitochondrial function and monoamine production. Deficiencies/insufficiencies of magnesium, Vitamin D, and B vitamins correlate with depression severity. Deficiencies of the folate and methylation cycles may lead to treatment-resistant depression. Imbalance of omega6 and omega-3 fatty acid intake allows more pro-inflammatory eicosanoids (prostaglandins, thromboxanes, leukotrienes) from omega- 6 than anti-inflammatory eicosanoids from omega-3. Refractory youth depression may be linked to abnormalities in functional biological systems, with excessive inflammation, oxidative stress, and gut-brain issues. Mediterranean diet, vitamins/minerals, omega-3 fatty acids, methyl donors, meditation, and exercise are worth considering as adjunctive treatments. More research is needed.

\section{Keywords}

Complimentary and Integrative psychiatry; depression; functional medicine; omega 3 fatty acids; adolescents; inflammation; oxidative stress; gut-brain axis; hypothalamicpituitary-adrenalaxis; diet; vitamin deficiencies

\section{Introduction}

Youth depression (ages 12-20) increased 37\% from 2004 to 2014 [1]. This coincided with a nine-fold increase in suicidal ideation in adolescents [2]. Suicide rates among females has increased by $65 \%$ since 2010 [3]. Some youth are refractory to antidepressants [4, 5]. Functional Medicine (which analyzes abnormalities in biologic functional systems) and Complementary and Integrative Medicine (CIM) interventions are being studied to address reasons for and interventions for treatment-refractory depression. Treatment refractory depression here is defined as any depression for which conventional/traditional treatments have not been successful.

Inflammation disrupts multiple pathways relevant to the risk of developing depression. Inflammation in the gut during stress, for instance, increases production of pro-inflammatory cytokines which stimulates the vagal nerve. The subsequent effect is that the hypothalamic-pituitary-adrenal (HPA) axis is upregulated, with adrenaline and cortisol increases. Sustained increases in cortisol and pro-inflammatory cytokines have been associated with depression [6]. Pro-inflammatory cytokines interfere with the indoleamine 2,3-dioxygenase (IDO) pathway, which decreases neurotransmitter production and disrupts microglia, leading to depressive symptoms [7]. Inflammation can also lead to oxidative stress which depletes glutathione needed to neutralize reactive oxygen species (ROS). The overabundance of ROS leads to mitochondrial dysfunction. Damage to the mitochondrial electron transport chain has been associated with depression and bipolar disorder [8]. Inflammation can also disrupt the folate and methylation cycles by decreasing the absorption of vitamin B12. B12 also plays a major role in neurotransmitter production (Figure 1).

Inflammation has been linked to obesity and diet. High-processed- fat and high-sugar foods cause dysbiosis or changes in the gut that cause inflammation and intestinal permeability that leads to insulin resistance and weight gain [9]. Processed foods, sugary beverages, and sedentary habits leading to 
weight gain are associated with a higher incidence of depression [10]. One study found that adolescents who were depressed had a $70 \%$ increased risk of being obese and obese adolescents had a $40 \%$ increased risk of being depressed [11].

Other modern factors that may relate to disruption of the microbiome and production of proinflammatory cytokines include pesticides [12], increased stress [6], and antibiotics [13].

Functional Medicine has begun to recognize abnormalities in biological systems that may prevent full recovery from depression. Complementary and Integrative Medicine (CIM) interventions to address these abnormalities may be used adjunctively.

Background Physiology/Immunology of Refractory Depression (Figure 1):

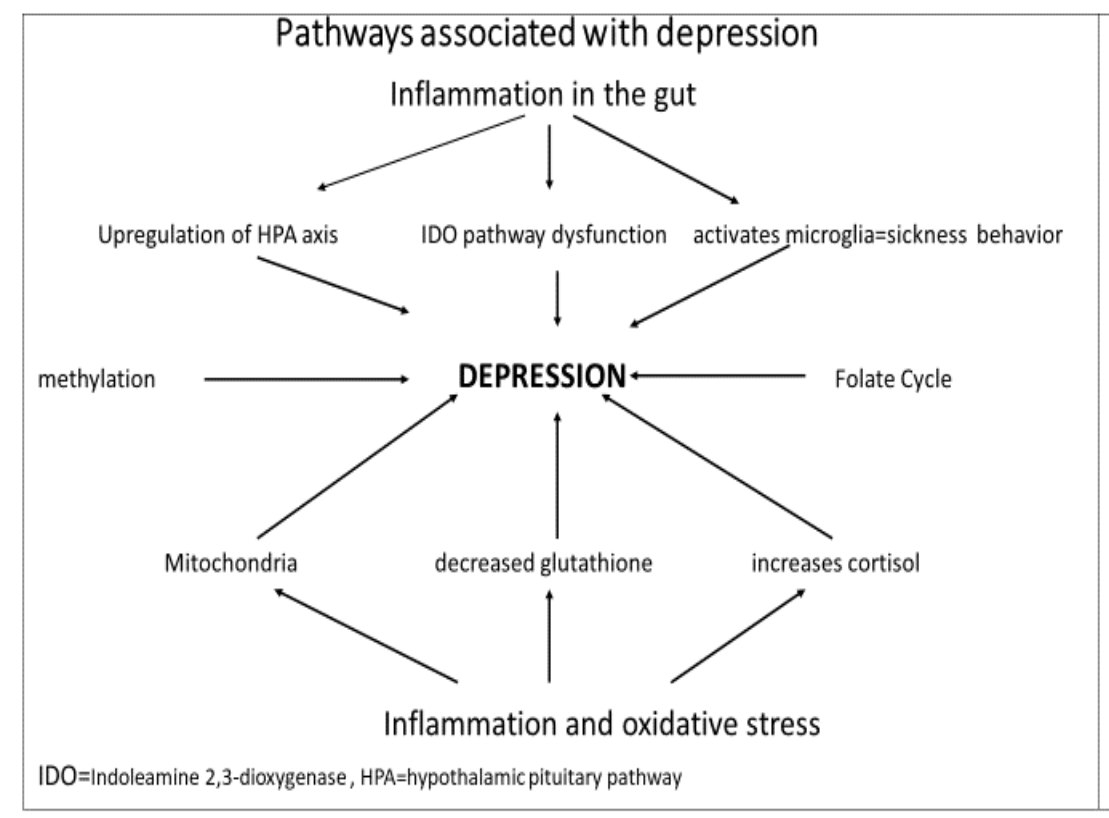

Figure 1

\subsection{Gut-Brain Axis}

A number of articles document dysbiosis-caused production of pro-inflammatory cytokines in the gut leading to psychiatric symptoms associated with depression $[6,14,15]$. In one study norepinephrine (induced by stress) caused an increase in E. coli [16] and decreases in Lactobacillus and Bifidobacterium, leading to increased lipopolysaccharides (LPS) [14]. LPS stimulate lymphocyte differentiation, leading to production of pro-inflammatory interleukin -beta (IL- $\beta$ ), interleukin-6 (IL-6), and tumor necrosis factor alpha (TNF $\alpha$ ) [17]. TNF $\alpha$ compromises mucosal integrity by promoting disintegration of tightjunction proteins, like occludin, in the gut wall. Pro-inflammatory cytokines also decrease short chain fatty acids (SCFA), like butyrate, acetate and propionate, responsible for maintaining gut integrity [15, 18]. Hence dysbiosis can lead to "leaky gut" $[6,7,15,18]$. Leaky gut leads to a corresponding breakdown of the blood-brain barrier, which allows inflammatory cytokines to enter the brain. Leaky gut can increase the risk for developing depressive symptoms in three ways: 
First, IL- 6 and TNF $\alpha$ stimulate the afferent fibers of the vagal nerve, which in turn stimulates the hypothalamic-pituitary-adrenal (HPA) axis. Normally this would cause a rise in norepinephrine and cortisol and cortisol would signal the HPA axis to normalize. During chronic stress with dysbiosis, the normal negative feedback loop from glucocorticoid is impaired $[15,18]$. The release of CRF from the hypothalamus is further potentiated by the circulating pro-inflammatory cytokines. Hence, the HPA axis becomes hyperactive, a frequent finding in patients with depression (Figure 1). Pro-inflammatory cytokines have been associated with anhedonia in adolescents [19]. In a study where adolescents collected saliva at waking and 30-minutes past waking for 3 days, greater cortisol awakening response (CAR) predicted greater depressive symptoms, and interacted with acute interpersonal stress in predicting depressive symptoms [20].

Second, "Sickness Behavior" (depressive symptoms) is associated with activation of microglia. Microglia can be activated by cytokines produced by macrophages and monocytes that cross the bloodbrain barrier, by increased levels of glutamate following acute stress exposure [21], and by increased gut LPS stimulating the afferent vagal nerve [22]. In turn, excess microglia stimulation can lead to excess cytokines (IL-beta and TNF alpha) which impair synaptic plasticity. The result is neuronal dysfunction with neuro-vegetative symptoms similar to depression (Figure 1) [23].

Third, increased proinflammatory cytokines activate Indoleamine 2,3-dioxygenase (IDO). IDO and its subsequent enzyme kynurenine monooxygenase divert tryptophan from serotonin production towards kynurenine, 3-hydroxykynurenine and subsequent quinolinic acid, a strong agonist of the glutamatergic $\mathrm{N}$-methyl-d-aspartate receptor, which has been linked to depression. In addition, all three of these aforementioned substances are associated with oxidative damage, inflammation, mitochondrial dysfunction, neurotoxicity, and lowered neuroplasticity [24]. Activation of IDO, measured by kynurenine-to-tryptophan ratio, correlates with severity of depressive symptoms [25] (Figures 1 and Figure 2).

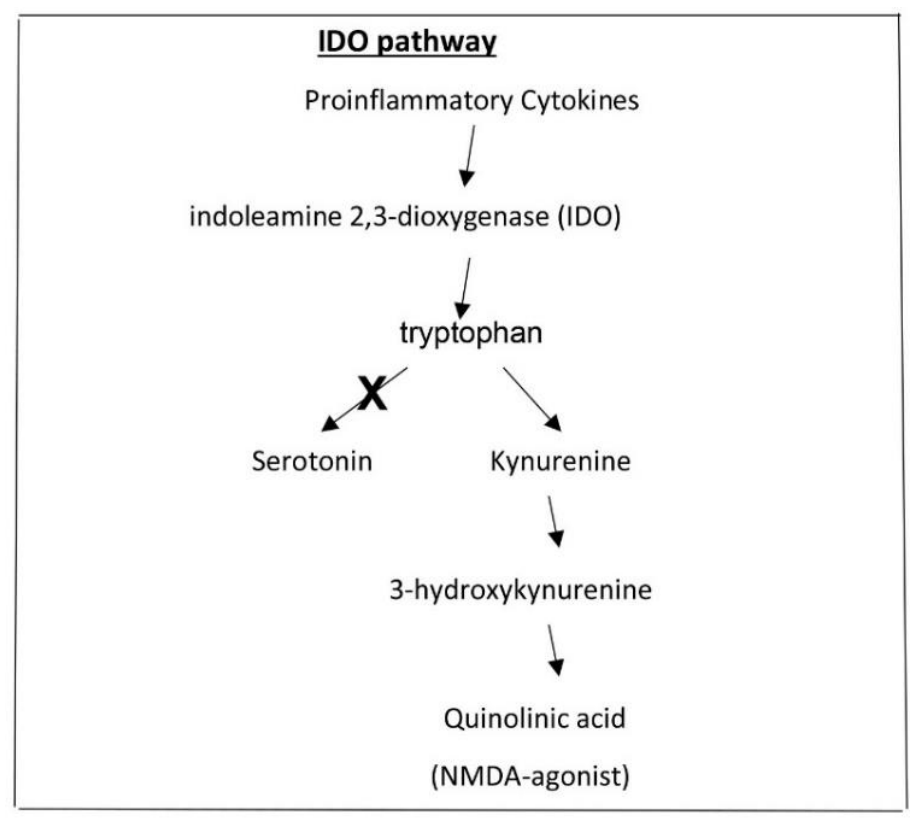

Figure 2 


\subsection{Inflammation, Oxidative Stress and Glutathione}

Inflammation or production of inflammatory cytokines can lead to oxidative stress. Oxidative stress involves the formation of Reactive Oxygen Species (ROS) or free radicals. Mitochondria can normally neutralize these free radicals [26]. However, when prolonged inflammation occurs, as in active mania, the antioxidant glutathione, is decreased resulting in tissue damage [27]. Reduced glutathione (GSH) is sacrificed by glutathione peroxidase to the oxidized disulfide form (GSSH) to neutralize ROS (See Figures 1 and Figure 3.) Mitochondrial dysfunction (Figure 1) results when the mitochondria are overwhelmed with the production of ROS and this results in damage to the mitochondrial electron transport chain, which has been associated with depression and bipolar disorder [28].

\section{Glutathione Conjugation}

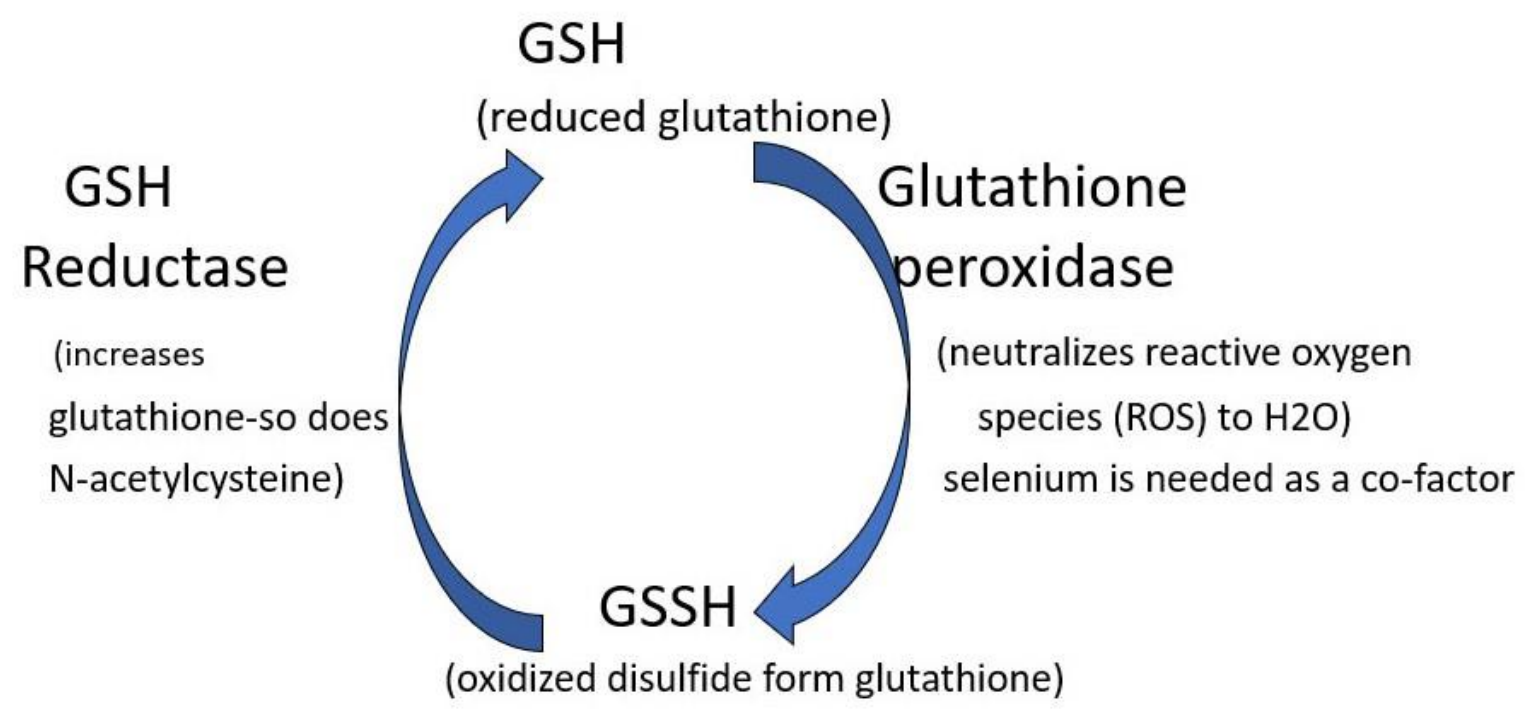

- Anti-oxidant molecules include CoQ10, alpha lipoic acid and primarily glutathione (increased with NAC) (Salim, 2015, Vavakova, 2015)

Figure 3

\subsection{B12 In the Folate and Methylation Cycles and the Effect of MTHFR Variations; Role of Gut Inflammation}

Inflammation in the gut can prevent the absorption of vitamin B12 and folate [29, 30]. This can lead to a corresponding decrease in S-adenosylmethionine (SAMe) and increased homocysteine (Figure 3). High homocysteine levels have been associated with depression [31]. If the methylene tetrahydrofolate reductase (MTHFR) gene $\mathrm{C677T}$ encodes a polymorphism variant of $\mathrm{C} / \mathrm{T}, \mathrm{T} / \mathrm{T}$ or $\mathrm{A} / \mathrm{C}$, a lower proportion of dietary folate is converted to L-methylfolate in the folate cycle and less homocysteine is utilized to produce SAMe with a corresponding decrease in neurotransmitter production, possibly leading to 
depression (Figure 4). In children with a history of childhood trauma, the MTHFR T/T genotype carriers developed a more severe form of depression [32].

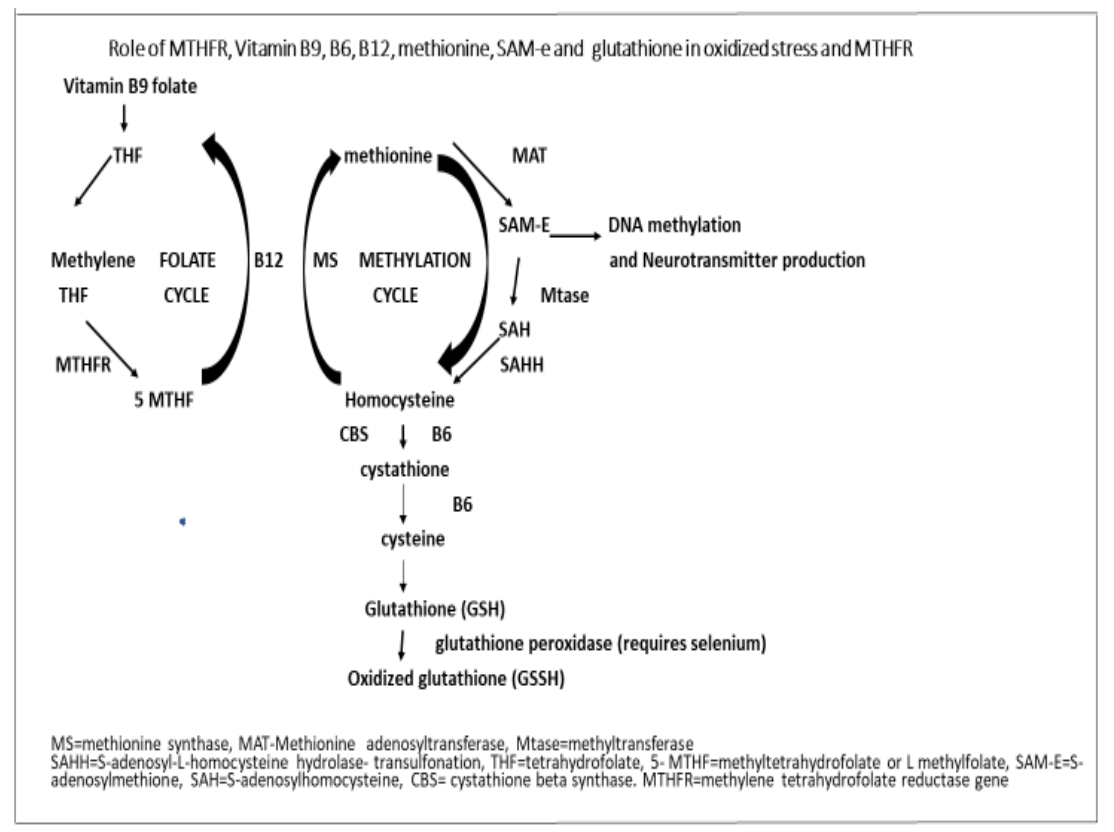

Figure 4

Thus, disturbances in the methylation cycle (by the siphoning off of glutathione to reduce oxidative stress) and in the folate cycle (by less absorption of Vitamin B12 and folate due to gut inflammation can lead to depression, which can be exacerbated by effects of the MTHFR genotype with a T allele (Figure 1).

\subsection{Lifestyle/Experience Contributions to Inflammation (Figure 5).}

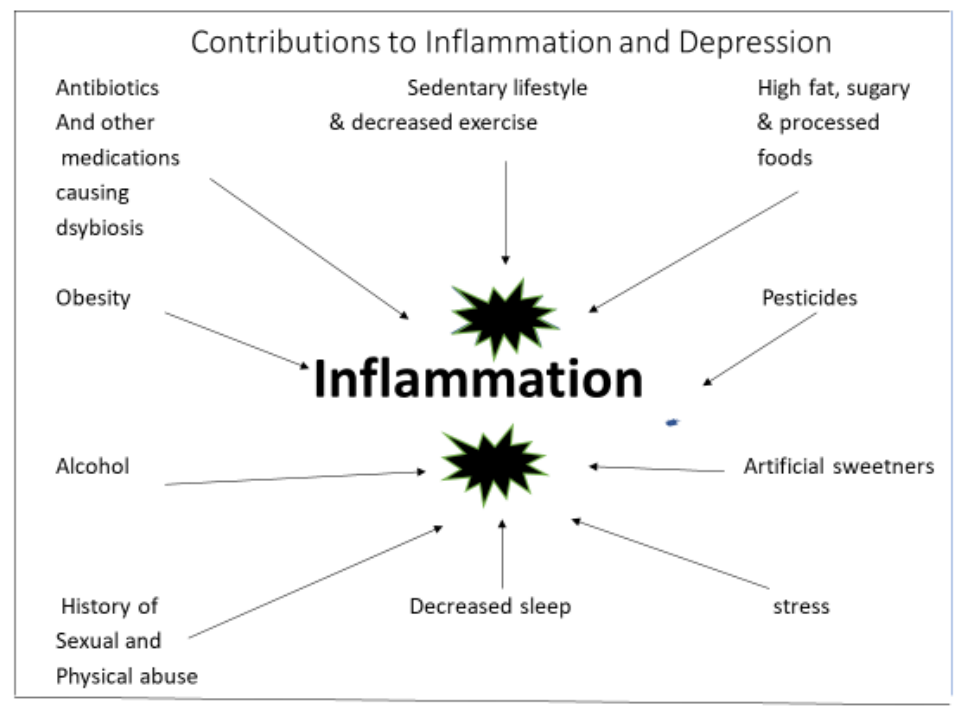

Figure 5 
The usual American diet may cause dysbiosis. High-fat and high-sugar fast foods and processed food can increase LPS, leading to dysbiosis and increase of intestinal permeability and depression symptoms [33]. Processed foods, sugary beverages, sedentary lifestyle, and associated weight gain are associated with higher incidence of depression [9, 10]. In addition, obesity from processed foods has been associated with depression [6, 11, 34]. In fact, high fat foods, processed foods and sugar interfere with leptin and insulin signaling. Decreased leptin signaling impairs satiety and increases insulin resistance leading to obesity and subsequent depression [35]. The use of artificial sweeteners to avoid sugar are linked to glucose intolerance $[29,36]$, may raise blood sugar, and favoring growth of bacteria associated with obesity, like Bacteroidetes and Firmicutes [29].

However, an inflammatory diet may be indicator of risk for depression regardless of high body mass index. In a study of the effects of an inflammatory diet and depression in children, data was prospectively collected over 10 years from the Avon Longitudinal Study of Parents and Children (ALSPAC) cohort $(\mathrm{N}=6939)$ free from depression at baseline (age 8.5 years). An inflammatory pattern (IDP) score from a food frequency questionnaire was measured at 8.5 years and levels of inflammatory biomarkers, C-reactive protein and interleukin-6, at 9.5 years. At age 18 years, depression cases were diagnosed via the International Statistical Classification of Diseases, 10th Revision (ICD-10) and the Clinical Interview Schedule-Revised (CIS-R) depression score. Participants in the highest tertile of IDP score had 1.34 times the risk of developing depression compared to those in the lowest tertile $(95 \% \mathrm{Cl}$, 1.08-1.66; $P$-trend<0.01). In addition, the correlation of IDP tertiles and depression showed a marginal trend among participants who were not overweight or obese $(p<0.10)$ but not among participants who were overweight or obese [37]. Therefore, inflammatory diets may also increase the risk of depression in non-obese children. Levels of IL-6 and CRP were measured in non-fasting blood samples obtained in participants at age 9 years in this study [38]. Participants in the top third of IL-6 values compared with the bottom third at age 9 years were more likely to be depressed (CIS-R) at age 18 years (adjusted odds ratio [OR], 1.55; 95\% Cl, 1.13-2.14). Results using the Mood and Feelings Questionnaire (MFQ) were similar. Risks of psychotic events and of psychotic disorder at age 18 years were also increased with higher IL-6 levels at baseline (adjusted OR, 1.81; 95\% Cl, 1.01-3.28; and 2.40; 95\% Cl, 0.88-6.22, respectively). Thus, later interventions may be helpful but may not be able to remedy inflammation completely. Early intervention should be the gold standard to avoid inflammation and risk for depression and other psychiatric disorders.

The reduction in the American diet of fermented food (containing probiotics) has also decreased the bioavailability of mood-regulating B vitamins, magnesium, and zinc, (which are necessary for the production of neurotransmitters) and these deficits are linked to depression [29, 30].

Exposure to sexual and physical abuse during childhood as well as sedentary lifestyle increases the risk of severe obesity [39, 40]. Insufficient sleep can also increase obesity by decreasing glucose tolerance, insulin sensitivity, and leptin and increasing ghrelin [41, 42]. Decreased sleep can increase cortisol hyperarousal and inflammation. Adolescents and young adults (age 10-22) with insomnia symptoms and short sleep duration ( $<7$ hours) had greater plasma c-reactive protein (CRP) levels than adolescents with insomnia symptoms and normal sleep duration ( $>7$ hours) [43].

Adolescents often begin experimenting with alcohol in their teens and some become alcoholdependent. One study demonstrated that some alcohol-dependent subjects developed gut "leakiness", 
associated with altered composition and activity of the gut microbiota and higher scores of depression, anxiety, and alcohol craving upon three weeks of abstinence [44].

Other modern factors that may lead to production of pro-inflammatory cytokines and increased gut permeability include pesticides [12]. Slow metabolizers (e.g., cytochrome 2D6) may not be able to metabolize pesticides, allowing them to accumulate to toxic levels causing inflammation and oxidative stress. Gut bacteria could be affected by pesticides, similar to certain medications like antibiotics [13]. In fact, in a study of over 2000 cases with depression (age 15-65), treatment with a single antibiotic course was associated with higher risk for depression, e.g., adjusted odds ratios were 1.23 for penicillins (95\% Cl, 1.18-1.29) and $1.25(95 \% \mathrm{Cl}, 1.15-1.35)$ for quinolones [45]. Proton pump inhibitors (PPI) and antipsychotic medications are associated with a decrease in diversity of gut microbiome and increased susceptibility to Clostridium difficile infection [46]. One study revealed a decreased ratio of Bacteroidetes: Firmicutes in the gut microbiome shortly after chronic use of risperidone in children, with a corresponding increase in body mass index over time [47].

Thus, inflammation caused by the change in the American diet to high fat, sugary, and processed foods, obesity, sedentary lifestyle, lack of sleep, stress, a history of sexual and physical abuse, alcohol dependence, artificial sweeteners, pesticides, and medications, can all play a role in the disruption of the gut-brain axis and subsequent development of depression, and may be linked to refractory depression (See Figure 5).

\subsection{Treatment Strategies}

Obviously, simple (but hard to implement) changes in lifestyle and diet may help avoid or decrease depression.

\subsection{Treatments Addressing Food and Vitamin/Mineral Deficiencies}

Moving to fermented foods (containing probiotics) can increase the bioavailability of moodregulating $B$ vitamins. Assessing magnesium, vitamin $D$, selenium, and zinc levels and supplementing as indicated may also be helpful. A number of studies in adolescents have linked depression to deficiencies in these micronutrients.

\subsubsection{Magnesium}

Magnesium deficiency is linked to a dysregulation of the HPA axis and increased inflammation. Magnesium modulates mood by acting as an N-methyl-d-aspartate (NMDA) antagonist, supporting synthesis of serotonin, dopamine and noradrenalin, increasing Brain Derived Neurotrophic Factor (BDNF) expression, and improving the sleep-wake cycle. Magnesium is found in green leafy vegetables, some legumes, seeds, nuts, and whole grains [48]. One study of 180 pregnant adolescents found higher symptoms of depression in those with lower magnesium intake [49].

Since inadequate brain magnesium appears to reduce serotonin levels, and since anti-depressants have been shown to raise brain magnesium, magnesium supplementation or eating fermented foods that increase magnesium may be beneficial for depression [50]. 


\subsubsection{Folate (Vitamin B9)}

Folate (B9), after its conversion to L-methyl folate) is a co-factor for biosynthesis of SAMe. The latter is involved in the production of norepinephrine, dopamine and serotonin [51] (Figure 4). Foods containing vitamin B9 include lettuce, asparagus, spinach, broccoli, and oranges [31]. A study of 26 inpatient girls admitted with depression found serum levels of docosahexaenoic acid (DHA), arachidonic acid ( $A A)$, and folate significantly lower than those in a control group [52]. A cross-sectional study of over 6000 adolescents found folate intake inversely associated with depressive symptoms in both boys and girls [53]. In a study of ten adolescents with treatment-resistant depression (mean age $14.4 \pm 2.8$ years), 8 (80\%) had a single mutation among the 2-methylene tetrahydrofolate reductase (MTHFR) gene variants evaluated (50\% A1298 AC; 30\% C677 CT), indicating reduced MTHFR activity. All had failed 3 antidepressant trials. When the 10 were prescribed adjunctive I-methylfolate (LM), 8 ( $80 \%)$ demonstrated improvement in depression, anxiety, and irritability [54].

\subsubsection{Cobalamin (Vitamin B12) (Figure 4)}

Like vitamin B9, B12 is involved in the synthesis of neurotransmitters. Considering the relationship between declining absorption of B12 and increasing depression in the elderly, it may be helpful to monitor B12 in youth with chronic malabsorption, like Celiac disease. Vitamin B12 is found in animal protein, such as, liver, salmon, yogurt and eggs [48]. A youth with severe B12 deficiency, depression, and psychotic symptoms demonstrated improvement 1-2 weeks after B12 injections [55].

\subsubsection{Pyroxidine (Vitamin B6) (Figure 4)}

Vitamin B6 is involved in the production of glutathione (Figure 2). It is found in poultry, fish, vegetables, eggs, and brown rice [48]. A cross-sectional study in 3,067 boys and 3,450 girls aged 12-15 found B-6 intake was inversely associated with depressive symptoms (score $\geq 16$ on Center for Epidemiologic Studies Depression Scale) in both boys (OR $[95 \% \mathrm{Cl}], 0.73[0.54,0.98]$; $p$ for trend $=.02$ ) and girls (OR [95\% Cl], $0.72[0.56,0.92]$; p for trend $=.002)$ [53].

\subsubsection{Zinc}

Zinc is a cofactor for $>100$ enzymes. Its deficiency results in increased glutamate, an excitatory molecule found in depression. Glutamate affects the HPA axis, increasing cortisol. Zinc acts as an antidepressant by antagonizing NMDA receptors and elevating BDNF in the hippocampus and cortical regions. Zinc is found in beans, nuts, red meat, oysters, crabs, lobsters, whole grains, and dairy products [48].

In a meta-analysis involving 17 studies with 1643 depressed and 804 control subjects (including young adults), peripheral blood zinc concentrations were approximately $1.85 \mu \mathrm{mol} / \mathrm{L}$ lower in depressed subjects than controls. Depression was associated with a lower concentration of zinc in peripheral blood [49]. Zinc has been used successfully for augmentation in previously treatment- 
resistant adults with depression and plays a role in producing neurotransmitters by helping convert dietary pyridoxine to the active form of B6, pyridoxal phosphate [50].

\subsubsection{Calciferol (Vitamin D3)}

In depression, 25(OH)D level was inversely associated with symptom severity, suggesting a doseresponse gradient, and low vitamin $\mathrm{D}$ carried risk of having a depressive disorder at 2-year follow-up [39]. Foods containing Vitamin D are eggs, liver, salmon, milk (artificially added), oysters and shrimp but most vitamin D comes from the sun [48]. Vitamin D 3 deficiency has been linked to increasing levels of pro-inflammatory cytokines and insulin resistance [30]. Vitamin D is considered a neurohormone essential to brain development, and deficiencies have been linked to depression, bipolar disorder, schizophrenia, and autism $[51,56,57]$. A prospective study assessed Vitamin D levels and a Mood and Feelings Questionnaire in 2,750 youth (mean ages $9.8,10.6$, and 13.8 years). Higher levels of Vitamin D at 9.8 years predicted lower depressive symptoms at 13.8 years but not at 10.6 years, suggesting that puberty brings more sensitivity to this deficiency [58]. An open label study treated 48 adolescents with clinical depression and low vitamin D levels for 3 months (4000 IU/ day for one month, then $2000 \mathrm{IU} /$ day for 2 months). The youth demonstrated improvements in self-rated depression scores $(p<.05)$ on the Mood and Feelings Questionnaire-short version [59]; without a control group, it is difficult to interpret this result.

Thirty-four 18-70-year olds with DSM IV bipolar depression and Vitamin D insufficiency ( $<30 \mathrm{ng} / \mathrm{ml}$ ) were randomized to 5000 IU Vitamin D3/day vs. placebo for 12 weeks. Despite a greater rise in Vitamin $D$ levels in the supplemented group, there was no significant difference in reduction of depressive symptoms. However, both groups' Vitamin D levels remained insufficient [60]. Therefore, higher dosages and/or longer treatment may have improved symptoms. In fact, a cross-sectional study in adults found that the risk of depression only began to decrease when Vitamin $D$ levels were above 42 $\mathrm{ng} / \mathrm{ml}$ [61]. Therefore, depression treatment may require higher levels along the line of claims that optimum levels should be between $50-80 \mathrm{ng} / \mathrm{ml}$. In addition, whether vitamin $D$ is taken with a fatty meal to promote absorption is an important clinical management issue.

\subsubsection{Selenium}

Selenium deficiency is associated with thyroid dysregulation, oxidative stress, and inflammation. Selenium is necessary for the conversion of GSH to GSSH to neutralize ROS (Figure 3). Selenium and zinc are necessary for the conversion of thyroxine (T4) to active triiodothyronine (T3). Selenium acts as an anti-depressant by modulating serotonin, dopamine and adrenaline and attenuating inflammation. Foods containing selenium include seafood, grains, meat, poultry, fish and eggs. Where the food was grown affects Se levels because of wide variation in regional soil levels [48].

More than $50 \%$ of depressed pregnant teenagers had an inadequate intake (excluding dietary supplement) of folate, vitamin A, vitamin E, iron, zinc, calcium, magnesium, and phosphorous. Additionally, $>20 \%$ of participants had an inadequate intake of thiamin, riboflavin, niacin, vitamin B6, vitamin B12, vitamin C, copper, and selenium [49]. 
In summary, Zinc, magnesium, B-vitamin, selenium, and Vitamin-D deficiencies should be considered in depressed youth. Blood levels should be monitored in unresponsive cases. Also, measuring deficits in the folate cycle like folate, vitamin B12 or methylmalonic acid (an early indicator of B12 deficiency), and checking MTHFR genotype may be helpful. Measuring deficiencies in the methylation cycle may also be helpful, which would include homocysteine, SAM-E and methylmalonic acid (Figure 3). A broadspectrum micronutrient supplement may be the most practical way to address the majority of the deficiencies/insufficiencies.

\subsection{Treatment for Oxidative Stress}

Lower levels of total and reduced glutathione (GSH) have been associated with later onset of bipolar disorder (BD). Although a low GSH/GSSH ratio level may be indicative of oxidative stress, a better indicator of oxidative stress, which damages mitochondria, may be serum superoxide dismutase (SOD). An increase in SOD, which neutralizes ROS found in oxidative stress, has been associated with BD [27]. SOD was significantly increased $(p<0.001)$ in manic and depressed BD compared to either controls or euthymic BD patients. The superoxide dismutase increase may be a compensatory mechanism in the acute phase of mania when oxidative stress is at its highest [62].

\subsection{N-Acetylcysteine (Nac)}

Administration of NAC, a cysteine prodrug, replenishes intracellular GSH levels. NAC is best known for its ability to counter acetaminophen (Tylenol) toxicity, and is a safe, well-tolerated antidote for cysteine/GSH deficiency. NAC is known to decrease oxidative stress by increasing glutathione and it modulates glutamate [63]. Oxidative stress and glutamate transmission are involved in both depression and Cannabis Use Disorder (CUD). Secondary analyses in an 8-week randomized controlled trial of Nacetylcysteine (NAC) for cannabis cessation found that the 74 adolescents with baseline depressive symptoms had significantly fewer positive urine cannabinoid tests than those without depressive symptoms. [64]. (see Figure 3). Perhaps decreasing oxidative stress indirectly influenced depression which may have decreased the urge to self-medicate with cannabis. In an RCT of adult bipolar patients $1000 \mathrm{mg}$ of NAC twice a day resulted in a large decrease in depressive symptoms compared to the placebo group [65]. NAC seems to do more for BD depression than for mania.

\subsection{Treatment to Address Inflammation}

\subsubsection{Essential Fatty Acids}

Both omega-6 $(\Omega 6)$ and omega-3 $(\Omega 3)$ fatty acids are considered essential; they are precursors of pro-inflammatory and anti-inflammatory eicosanoids (prostaglandins, leukotrienes, thromoboxanes), respectively. In the $\Omega 3$ series, eicosapentaenoic acid (EPA) provides anti-inflammatory cell signalers while docosahexaenoic acid (DHA) (and to some extent EPA) is needed for neuronal membranes. The balance of $\Omega 6: \Omega 3$ provides a "supply side" regulation of inflammation. Youth with mood disorders have higher ratios of omega-6 $(\Omega 6)$ to omega-3 fatty acids $(\Omega 3)$ than those without mood disorders. In a 
seven-year follow-up of youth with a high risk for psychosis, a higher $\Omega 6: \Omega 3$ ratio at baseline predicted later mood disorders but not other psychiatric disorders [66].

A randomized pilot trial $(\mathrm{N}=23$ ) in 5-12-year old children found greater reduction of depressive and manic symptoms with a combination of $\Omega 3$ fatty acids and inositol than with inositol alone [67]. In 28 children with major depression, $70 \%$ of those receiving $\Omega 3$ at a 2:1 ratio of EPA:DHA [380-400 mg EPA; 180-200 mg DHA) but none receiving placebo enjoyed greater than $50 \%$ reduction in depressive symptoms. In a randomized comparison of $\Omega 6$ to $\Omega 3$ supplementation for depression in 11-17-yearolds, only omega-3 showed significant improvement, and this was confined to those with major depression rather than mixed anxiety \& depression [68].

In the Omega-3 and Therapy Studies (OATS), 23 youth age 8-14 with bipolar disorder NOS and 72 with depression were assigned in a $2 \times 2$ design to $\Omega 3$ (1.6 g/day of EPA+DHA), psychoeducational psychotherapy (PEP) plus placebo matched to $\Omega 3, \Omega 3$ plus PEP, or placebo. In the bipolar study $\Omega 3$ showed more benefit for depression than placebo, both alone $(d=.48)$ and combined with psychoeducational psychotherapy (PEP) $(d=1.7, p=.01)$ [69]. In the depression study, $\Omega 3$ alone $(d=.42)$ and combined with PEP $(d=.28)$ surpassed placebo in relief of depressive symptoms, but not significantly. However, fewer psychosocial stressors significantly moderated a more favorable depression response to $\Omega 3$, suggesting value for more biologically based depression rather than reactive depression. [70]. Also, those with higher BMI benefitted significantly more from $\Omega 3$ compared to placebo than those with lower BMI [71]. In the same two studies, an analysis of the pooled samples found significantly greater $(p=0.001, d=0.70)$ improvement with $\Omega 3$ than placebo on a parent-rated composite scale of executive function [72].

Considering all available data, supplementation with up to a gram per day of mercury-free EPA+DHA seems advisable in treating child or adolescent depression. For safety and optimal utilization, antioxidant vitamins/minerals (possibly as broad spectrum micronutrients) should be given concomitantly.

\subsubsection{Probiotics}

Probiotic treatment has been shown to reduce the hyperactivity of the HPA axis [73]. In an RCT 66 patients hospitalized with mania were adjunctively given either Lactobacillus rhamnosus strain GG and Bifidobacterium animalis subsp. lactis strain $\mathrm{Bb} 12$ or placebo in a parallel two-group design. During the 24-week observation period there were a total of 24 rehospitalizations in the 33 individuals who received placebo and eight rehospitalizations in the 33 individuals who received the probiotics $(z=2.63$, $P=.009$ ) [74]. Another study, [75] found that probiotic formulations of Bifidobacterium longum and Lactobacillus helveticus significantly decreased anxiety in humans. In a double-blind RCT [76] 40 adults with a DSM-IV diagnosis of MDD age 20-55 had significant beneficial effects on Beck Depression Inventory, insulin resistance, hs-CRP concentrations, and glutathione concentrations with Lactobacillus acidophilus, $2 \times 10(9)$ Colony-Forming Units (CFU)/g, Lactobacillus casei $2 \times 10(9)$ CFU/g), and Bifidobacterium bifidum $2 \times 10$ (9) CFU/g for 8 weeks.

Combining probiotics with prebiotics seems even more beneficial. Prebiotics can lower LPS and increase satiety peptides (thus decreasing hunger and metabolic parameters associated with obesity) 
[77]. Probiotics (Lactobacilli and Bifidobacterium) with prebiotics improved body weight, abdominal fat, and intestinal barrier function [78]. In a randomized trial [79] 110 depressed patients were randomly assigned to receive the probiotic (Lactobacillus helveticus and Bifidobacterium longum), prebiotic (galactooligosaccharide), or placebo for 8 weeks. Eighty one patients completed the study. Probiotic supplementation resulted in a significant decrease in BDI score (17.39 to 9.1) compared to the placebo (18.18 to 15.55$)$ and prebiotic $(19.72$ to 14.14$)$ supplementation $(p=0.042)$. The kynurenine/tryptophan ratio (an indicator of the IDO pathway) decreased significantly in the probiotic group compared to placebo after adjusting for serum isoleucine $(p=0.048)$.

Probiotics may decrease depression by decreasing inflammation and oxidative stress. In a metaanalysis probiotics supplementation resulted in significant reduction in the Hamilton Depression Rating Scale (HAMD) and a reduction in C-reactive protein, IL-10, and malondialdehyde (an indicator of oxidative stress). However, changes in the Beck Depression Inventory, TNF- $\alpha$, IL-6, nitric oxide (indicator of free radicals), glutathione, and total antioxidant capacity [80] were not significant.

Early interventions to change the course of dysbiosis may be helpful. Probiotic feeding during the early years modulated the gut microbiota 10 years later, which restrained excessive weight gain [81]. In a RCT, 75 infants were randomized to Lactobacillus rhamnosus or placebo during the first 6 months of life and were followed for 13 years. Gut microbiota was assessed at the ages of $3 \mathrm{wk}, 3,6,12,18,24$ mo, and 13 years. ADHD or autism was diagnosed in $6 / 35$ (17.1\%) of children in the placebo group and none in the probiotic group $(P=0.008)$. The mean $(S D)$ numbers of Bifidobacterium species bacteria in feces had been lower during the first 6 mo of life in affected children 8.26 (1.24) log cells/g than in healthy children $9.12(0.64) \log$ cells $/ g ; P=0.03$. Probiotics fed to infants decreased intestinal permeability as measured by lactulose/mannitol ratio [82]. Prospective larger studies are needed to determine if early use of probiotics may alleviate the risk of developing depression associated with "leaky gut".

\subsection{Supplements (Other Than Vitamins or Minerals) and Lifestyle Interventions that May Affect Inflammation, the Methylation Cycle, Folate Cycle and Cortisol Levels}

\subsubsection{Saffron}

Saffron has effects similar to anti-depressants via serotonin effects. It also has anti-inflammatory and anti-oxidant effects. In a meta-analysis, consumption of saffron resulted in a significant reduction in Beck Depression Inventory (BDI) (11 studies --weighted mean difference (WMD): -4.86; 95\% Cl: -6.58, -3.14), Beck Anxiety Inventory (BAI) (5 studies) (WMD: -5.29; $95 \% \mathrm{Cl}:-8.27,-2.31$ ) and Pittsburgh Sleep Quality Index (PSQI) scores (3 studies) (WMD: -2.22; $95 \% \mathrm{Cl}:-2.73$, -1.72). It did not decrease C-reactive protein levels [83].

\subsubsection{Adaptogens}

Adaptogens are herbs used in Ayurvedic and Chinese medicine. They reduce oxidative stress by reducing the levels of nitrous oxide and help to normalize an upregulated HPA axis by unblocking the glucocorticoid receptor so that the normal homeostatic feedback process can occur [84]. 
In a RCT Eleutherococcus senticosus and lithium were compared to fluoxetine and lithium in 76 adolescents ages $12-17$ with bipolar depression. Remission rates $(51.4 \%$ vs $48.7 \%$ and response rates (67.6\% vs $71.8 \%$ ) were comparable for E senticosus vs fluoxetine. Three of the adolescents on fluoxetine experienced a manic switch [85].

\subsubsection{St John's Wort (hypericum; SJW)}

St. John's Wort is known to modulate neurotransmitter levels and their receptors, including serotonin, norepinephrine, dopamine, $\mathrm{y}$-aminobutyric acid, and glutamate; modify inflammatory cytokines; inhibit cortisol production; modulate neuronal ionic conductance; elevate intracellular sodium concentration; and induce neurogenesis and neuroprotection. However, in the U.S. it is known to contain highly varied amounts of the active ingredient hypericum [86]. Batch-to-batch variability complicates reliable effects.

In an open trial [87] 33 youth with moderate to severe mood disorder (Child Depression Rating ScaleRevised (CDRS-R) >40) took $150-300 \mathrm{mg} 3$ times/day. Response criteria (CDRS-R <28) were met by $24 \%$ by week 1 and $83 \%$ by week 8 . Another open label trial [88] using $300 \mathrm{mg} 3$ times/day had similar results but there was a $57 \%$ drop out rate due to side effects.

Without a control group, the results are hard to interpret but the rapid response rate and positive results suggests more studies are needed. However, clinicians should be aware that St John's Wort can cause serotonin syndrome, discontinuation syndrome, and sunlight sensitivity and induce cytochrome P450 isoenzymes CYP 3A4, 2C19 and 2C9. There is also concern that at high dosages it may cause seizures and mania [89].

\subsubsection{S-adenosylmethionine (SAMe)}

Due to the fact that SAMe is difficult to synthesize and breaks down easily when exposed to water, some formulations may not have detectable levels. SAMe plays a role in the methylation cycle and is involved in neurotransmitter production (See Figure 3). An open trial in 3 youth with clinical depression using $600-1400 \mathrm{mg} /$ day found all had a rapid response that persisted for 22 weeks [90]. Clinicians should be aware that it can have the same effects as tricyclics and psychostimulants and can induce mania. It needs further study.

\subsubsection{Uridine}

Uridine is a pyrimidine nucleoside, thought to impact glutamate. Pyrimidines are thought to have beneficial effects on cerebral phospholipid metabolism, catecholamine synthesis, and mitochondrial function all of which have been linked to depression Seven bipolar adolescents treated with $500 \mathrm{mg} / \mathrm{d}$ for 6 weeks had significant decreases on the CDRS, from 65.6 to 27.2 [91]. It needs better study.

\subsubsection{Meditation}

Meditation may help normalize the hyperactivation of the HPA axis by decreasing vagal nerve stimulation [92]. In a study using yoga and meditation for 3 months there were increases in anti- 
inflammatory cytokines like IL-10, decreases in the pro-inflammatory cytokine Interleukin-12, increases in Brain Derived Neurotrophic Factor (BDNF). BDNF is a key neurotrophin that promotes development, survival and plasticity of neurons in the central and peripheral nervous systems. There were clearly significant improvements in the Brief Symptom Inventory-18 scores, including the total score $(\mathrm{t} .33 /=$ 4.66, $p<0.0001)$ as well as each of the subscores which included depressive $(t .33 /=2.84, p<0.01)$, anxious (t.33/ = 4.22, p < 0.0001), and somatic (t.33/ = 4.66, p < 0.0001) symptoms [93]. In a study of 58 individuals (age 19-50 years old) with Major Depression diagnosed with DSM-5 criteria, participants were randomly assigned to Yoga and meditation or a control group for 12 weeks. There was a significant decrease [difference between means, $(95 \% \mathrm{CI})$ ] in Beck Depression Index-II score change in value, time 1 and time $2[-5.83(-7.27,-4.39), p<0.001]$ and significant increase in BDNF (ng/ml) $[5.48(3.50,7.46)$, $\mathrm{p}<0.001$ ] after Yoga Meditation Lifestyle Intervention (YMLI) compared to control group. Among the mind-body communicative biomarkers, there was a significant increase in circulating dehydroepiandrosterone (DHEAS-an indication of reduced inflammation) and sirtuin 1 (used in repair of DNA) and a significant decrease in circulating cortisol and IL-6 (a pro-inflammatory cytokine) after YMLI compared to yoga group at baseline level and control group. A total of 12 weeks YMLI showed improvement in cellular health biomarkers that included - significant decrease in 8 Oxo-2'deoxyguanosine ( $8 \mathrm{OH} 2 \mathrm{dG}$ - a marker of DNA damage); significant increase in total anti-oxidant capacity (TAC) and decrease in ROS (markers of oxidative stress); and significant increase in telomerase activity (marker of telomere attrition) compared to control group (all $p<0.05$ ) (Table 3). However, change in telomere length was not significant in both groups. Also, the control group showed significantly increased ROS and IL-6 levels compared to baseline ( $<0.001)$. As noted earlier an overactive HPA and increased cortisol is associated with depression. In this study, increased cortisol was associated with reductions in BDNF and increase in depression severity [94]

\subsubsection{Exercise}

Exercise can increase Peroxisome Proliferation Activated Receptor Gamma Coactivator 1 (PGC1 alpha) gene expression, which reduces synthesis and release of pro-inflammatory cytokines and decreases glutamatergic neurotoxicity [95]. The Depressed Adolescents Treated with Exercise (DATE) study randomized 30 non-medicated adolescents age 12-18 with DSM-IV-TR major depressive disorder. Thirty adolescents aged 12-18 years were randomized to either vigorous exercise (EXER) (>12 $\mathrm{kg} / \mathrm{kcal} /$ week $[\mathrm{KKW}]$ ) or a control stretching activity (<4 KKW) for 12 weeks. By week 12 , the exercise group had a $100 \%$ response rate ( $86 \%$ remission), whereas the stretch group response rate was $67 \%$ (50\% remission) $(p=.02)[96]$.

\section{Discussion/Conclusions}

CIM interventions are appealing to parents and youth because of the perception that they are safer and will avoid stigma and possibly the need to see a psychiatrist regularly. However, they are not without risks, especially if parents or adolescents do not alert a clinician about use of over-the-counter supplements. There are many drug-supplement interactions. Most supplements and herbs have not been reviewed by the FDA before marketing and do not have FDA-approved good manufacturing 
procedures in place. Products may vary in amounts of active ingredients, and patients often take supplements or herbs without knowing established recommended dosages. The purity of supplements has been examined by Consumerlabs.Com, where clinicians may obtain more information. However, clinicians must be aware that some companies pay sites to recommend their products. Clinicians need to be aware of patient use of supplements and expand their knowledge of commonly used CIM treatments, keeping in mind that herbs, if they work, are crude drugs without good quality control.

Research increasingly recognizes that there may be abnormalities and individual variations in biological systems involving the gut-brain axis, inflammation, oxidative stress, folate and methylation cycles and cortisol levels that prevent conventional established interventions from being universally effective. CIM research, although in its infancy, is beginning to recognize that certain supplements and other interventions used adjunctively, targeting systems associated with these abnormalities, may decrease depressive symptoms. Addressing problems with inflammation in the gut, oxidative stress, mineral and vitamin deficiencies, diet, abnormalities in the folate and methylation cycles, sleep, sedentary lifestyle, obesity, and stress may help reduce the severity of depression, particularly if conventional interventions have not worked. Avoiding medications that disrupt the gut microbiome or that decrease B12 and CoQ 10 (like metformin) or proton pump inhibitors (which decrease B12) may reduce the severity of depression. When such drugs cannot be avoided, prophylactic B12 supplement should be considered. Using supplements like St. John's Wort (to increase neurotransmitters, decrease cortisol, and modify inflammatory cytokines), adaptogens (which allow the HPA axis to normalize and reduce oxidative stress), and saffron (which affects serotonin and acts as an anti-oxidant and antiinflammatory) may act as adjuncts in the treatment of refractory depression. The best interventions seem to include the addition of omega 3 fatty acids. Normalizing the gut with probiotics may also prove to be useful. Much more research is needed. One thing is clear: conventional interventions do not always work, and when they don't, other interventions should be considered.

Although it is imperative to decrease inflammation, once the inflammatory process becomes severe nutritional interventions may no longer be effective. E.g., In a RCT of 1950 patients, a protocol-guided individualized nutritional support program was compared to a standard hospital food control group. Baseline measurement of C-reactive protein (CRP) divided the sample into low, moderate and high inflammation ( $<10 \mathrm{mg} / \mathrm{L}, 10-100 \mathrm{mg} / \mathrm{L}$ and $>100 \mathrm{mg} / \mathrm{l}$, respectively). Compared to the control group, patients receiving nutritional support showed a significant reduction in 30-day mortality except the subgroup with high baseline inflammation, which showed no benefit of nutritional support [97]. Therefore, waiting to treat after inflammation occurs may lose efficacy once inflammation becomes severe.

While awaiting further research, there are some things clinicians can reasonably apply now. Figure 6 and 7 suggests a therapeutic decision-flow algorithm reflecting the current state of knowledge. 


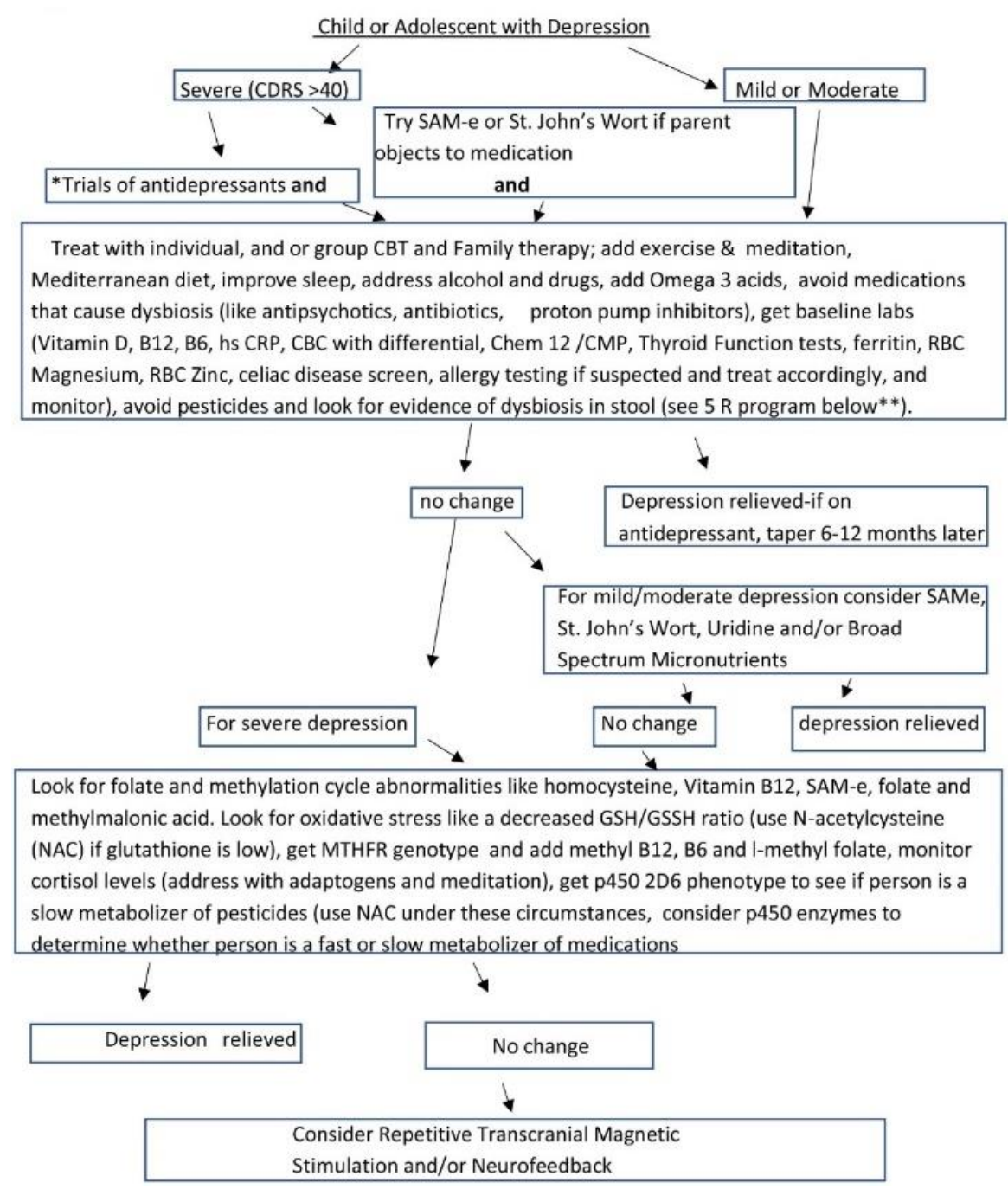

Figure 6

** 5 R's [98]

1. Remove-: allergic foods, parasites or other bad bugs such as bacteria or yeast. This might involve using an "elimination diet" to pick up sensitivities to certain foods

2. Replace: add back things like digestive enzymes, hydrochloric acid and bile acids that are required for proper digestion.

3. Reinoculate: Use probiotic foods or supplements that contain

bifidobacteria and lactobacillus species, and use high-soluble-fiber foods or prebiotic supplement that increases short chain fatty acids (SCFA) and maintains gut integrity.

4. Repair: Help repair the gut by adding key nutrients (like zinc) or antioxidants (like vitamins A, C, and E), L- glutamine and omega 3 fatty acids if not already added.

5. Rebalance: Pay attention to lifestyle choices - sleep, exercise and stress can all affect the GI tract. (See Figure 2)

Figure 7 [98] 


\section{Author Contributions}

Dr. Simkin was the author and Dr. Arnold was the co-author.

\section{Funding}

Dr. Simkin has no disclosures.

Dr. Arnold has received research funding from Curemark, Forest, Lilly, Neuropharm, Novartis, Noven, Shire, Supernus, Roche, Otsuka, and Young Living (as well as NIH and Autism Speaks), has consulted with Gowlings, Neuropharm, Organon, Pfizer, Sigma Tau, Shire, Tris Pharma, and Waypoint, and been on advisory boards for Arbor, Ironshore, Novartis, Noven, Otsuka, Pfizer, Roche, Seaside Therapeutics, Sigma Tau, Shire.

\section{Competing Interests}

The authors have declared that no competing interests exist.

\section{References}

1. Mojtabai R, Olfson M, Han B. National trends in the prevalence and treatment of depression in adolescents and young adults. Pediatrics. 2016; 138: e 20161878

2. Lara GA, Zuniga JO, Perez OC, Solís SH, Jiménez CE, Méndez MC. Predictors of suicidal ideation and depressive symptoms in Chiapas, Mexico. Cien Saude Colet. 2018; 23: 1089-1096.

3. Twenge JM, Joiner TE, Rogers ML, Martin GN. Increases in depressive symptoms, suicide-related outcomes, and suicide rates among U.S. Adolescents after 2010 and links to new media screen time. Clin Psychol Sci. 2018; 6: 13-17.

4. Cipriani A, Zhou X, Del Giovane C, Hetrick SE, Qin B, Whittington C, et al. Comparative efficacy and tolerability of antidepressants for major depressive disorder in children and adolescents: $A$ network meta-analysis. Lancet. 2016; 388: 881-890.

5. Ignaszewski MJ, Waslick B. Update on randomized placebo-controlled trials in the past decade for treatment of major depressive disorder in child and adolescent patients: A systematic review. J Child Adol Psychop. 2018; 28: 668-675.

6. Foster JA, Rinaman L, Cryan JF. Stress \& the gut-brain axis: Regulation by the microbiome. Neurobiol Stress. 2017; 7: 124-136.

7. Simkin DR. Microbiome and mental health, specifically as it relates to adolescents. Currt Psychiatry Rep. 2019; 21: 93.

8. Scaini G, Rezin GT, Carvalho AF, Streck EL, Berk M, Quevedo J. Mitochondrial dysfunction in bipolar disorder: Evidence, pathophysiology and translational implications. Neurosci Biobehav Rev. 2016; 68: 694-713.

9. Moreira AP, Texeira TF, Ferreira AB, Peluzio MD, Alfenas RD. Influence of a high-fat diet on gut microbiota, intestinal permeability and metabolic endotoxaemia. Br J Nutr. 2012; 108: 801-809. 
10. Malik VS, Willett WC, Hu FB. Sugar-sweetened beverages and BMI in children and adolescents: Reanalyses of a meta-analysis. Am J Clin Nutr. 2009; 89: 438-439.

11. Mannan M, Mamun A, Doi S, Clavarino A. Prospective associations between depression and obesity for adolescent males and females-a systemic review and meta-analysis of longitudinal studies. PloS One. 2016; 11: e0157240

12. Singh NK, Banerjee BD, Bale K, Basu M, Chhillar N. Polymorphism in cytochrome P450 2D6, glutathione S-transferases Pi 1 genes, and organochlorine pesticides in Alzheimer disease: A casecontrol study in north Indian population. J Geriatr Psychiatry Neurol. 2014; 27: 119-127.

13. Reijnders D, Goossens GH, Hermes GD, Neis EP, Van der Beek CM, Most J, et al. Effects of gut microbiota manipulation by antibiotics on host metabolism in obese humans: $A$ randomized double-blind placebo-controlled trial. Cell Metab. 2016; 24: 63-74.

14. Kelly JR, Kennedy PJ, Cryan JF, Dinan TG, Clarke G, Hyland NP, et al. Breaking down the barriers: The gut microbiome, intestinal permeability and stress-related psychiatric disorders. Front Cell Neurosci. 2015; 9: 392.

15. Bonaz B, Bazin T, Pellissier S. The vagus nerve at the Interface of the microbiota-gut-brain axis. Front Neurosci. 2018; 12: 49.

16. Lyte M, Bailey MT. Neuroendocrine-bacterial interactions in a neuro toxin induced model of trauma. J Surg Res. 1997; 70: 195-201.

17. Karlović D, Serretti A, Vrkić N, Martinac M, Marčinko D. Serum concentrations of CRP, IL-6, TNF- $\alpha$ and cortisol in major depressive disorder with melancholic or atypical features. Psychiatry Res. 2012; 198: 74-80.

18. Plöge S, Stumpff F, Penner GB, Schulzke JD, Gäbel G, Martens H. Microbial butyrate and its role for barrier functioning in the gastrointestinal tract. Ann N Y Acad Sci. 2012; 1258: 52-59.

19. Freed RD, Mehra LM, Laor D, Patel M, Alonso CM, Kim-Schulze S, et al. Anhedonia as a clinical correlate of Inflammation in psychiatric disorders. World J Biol Psychiatry. 2019; 20: 712-722.

20. Stroud CB, Vrshek-Shallhorn S, Norkett EM, Doane LD. The cortisol awakening response (CAR) interacts with acute interpersonal stress to prospectively predict depressive symptoms among early adolescent girls. Psychoneuroendocrinology. 2019; 107: 9-18.

21. Maes M, Berk M, Goehler L, Song C, Anderson G, Gałecki P, et al. Depression and sickness behavior are Janus-faced responses to shared inflammatory pathways. BMC Med. 2012; 10: 66.

22. Bi $W$, Zhu L, Jing $X$, Zeng $Z$, Liang $Y, X u A$, et al. Rifampicin improves neuronal apoptosis in LPSstimulated co-cultured BV2 cells through inhibition of the TLR-4 pathway. Mol Med Rep. 2014; 10: 1793-1799.

23. DiSabato DJ, Quan N, Godbout JP. Neuroinflammation: The devil is in the details. J Neurochem. 2016; 139: 136-153.

24. Barreto FS, Chaves Filho AJ, de Araújo MC, de Moraes MO, de Moraes ME, Maes M, et al. Tryptophan catabolites along the indoleamine 2,3-dioxygenase pathway as a biological link between depression and cancer. Behav Pharmacol. 2018; 29: 165-180.

25. Swardfager W, Herrmann N, Dowlati Y, Oh PI, Kiss A, Walker SE, et al. Indoleamine 2,3-dioxygenase activation and depressive symptoms in patients with coronary artery disease. Psychoneuroendocrinology. 2009; 34: 1560-1566. 
26. Murphy MP. Mitochondrial thiols in antioxidant protection and redox signaling: Distinct roles for glutathionylation and other thiol modifications. Antioxid Redox Signal. 2011; 16: 476-495.

27. Rosa AR, Singh N, Whitaker E, De Brito M, Lewis AM, Vieta E, et al. Altered plasma glutathione levels in bipolar disorder indicates higher oxidative stress; a possible risk factor for illness onset despite normal brain-derived neurotrophic factor (BDNF) levels. Psychol Med. 2014; 44: 2409-1418.

28. Rezin GT, Amboni G, Zugno Al, Quevedo J, Streck EL. Mitochondrial dysfunction and psychiatric disorders. Neurochem Res. 2009; 34: 1021.

29. Shell ER. Artificial sweeteners get a gut check. Sci Am. 2015; 31: 32-34.

30. Milaneschi Y, Hoogendijk W, Lips P, Heijboer AC, Schoevers R, Van Hemert AM, et al. The association between low vitamin D and depressive disorders. Mol Psychiatry. 2014; 19: 444-451.

31. Saraswathy KN, Ansari SN, Kaur G. Association of vitamin B12 mediated hyperhomocysteinemia with depression and anxiety disorder: A cross-sectional study among Bhil indigenous population of India. Clin Nutr ESPEN. 2019; 30: 199-203.

32. Lo A, Bockting CL, Koeter MW, Snieder H, Assies J, Mocking RJ, et al. Interaction between MTHFR C677T polymorphism and traumatic childhood events predicits depression. Tranl Psychiatry. 2013; 3: e288.

33. Sánchez-Villegas A, Toledo E, De Irala J, Ruiz-Canela M, Pla-Vidal J, Martínez-González MA. Fastfood and commercial baked goods consumption and the risk of depression. Public Health Nutr. 2012; 15: 424-432.

34. Costa CS, Del-Ponte B, Assuncao MC, Santos IS. Consumption of ultra-processed foods and body fat during childhood and adolescence: A systematic review. Public Health Nutr. 2018; 21: 148-159.

35. Mobbs CV, Isoda F, Makimura H, Mastaitis J, Mizuno T, Shu IW, et al. Impaired glucose signaling as a cause of obesity and the metabolic syndrome: The glucoadipostatic hypothesis. Physiol Behav. 2005; 85: 3-23.

36. Suez J, Korem T, Zeevi D, Zilberman-Schapira G, Thaiss CA, Maza O, et al. Artificial sweeteners induce glucose intolerance by altering the gut microbiota. Nature. 2014; 514: 181-186.

37. Cong X, Tracy M, Edmunds LS, Hosler AS, Appleton AA. The relationship between inflammatory dietary pattern in childhood and depression in early adulthood. Brain Behav Immun Health. 2020; 2: 100017.

38. Khandaker GM, Pearson RM, Zammit S, Lewis G, Jones PB. Association of serum interleukin 6 and C-reactive protein in childhood with depression and psychosis in young adult life: A populationbased longitudinal study. JAMA Psychiatry. 2014; 71: 1121-1128.

39. Richardson AS, Dietz WH, Gordon-Larsen P. The association between childhood sexual and physical abuse with incident adult severe obesity across 13 years of the national longitudinal study of adolescent health. Pediatr Obes. 2014; 9: 351-361.

40. Strasburger VC. Children, adolescents, obesity, and the media. Pediatrics. 2011; 128: 201-208.

41. Fatima Y, Doi SA, Mamun AA. Longitudinal impact of sleep on overweight and obesity in children and adolescents: A systematic review and bias-adjusted meta-analysis. Obes Rev. 2015; 16: 137149.

42. Pan W, Kastin AJ. Leptin: A biomarker for sleep disorders? Sleep Med Rev. 2014; 18: 283-290 
43. Fernandez-Mendoz J, Li Y, Vgontza A, Fang J, Gaines J, Calhoun SL, et al. Insomnia is associated with cortical hyperarousal as early as adolescence. Sleep. 2016; 39: 1029-1036.

44. Leclercq S, Matamoros S, Cani PD, Neyrinck AM, Jamar F, Stärkel P, et al. Intestinal permeability, gut-bacterial dysbiosis, and behavioral markers of alcohol-dependence severity. Proc Natl Acad Sci U S A. 2014; 111: E4485-4493.

45. Lurie I, Yang YX, Haynes K, Mamtani R, Boursi B. Antibiotic exposure and the risk for depression, anxiety, or psychosis: A nested case-control study. J Clin Psychiatry. 2015; 76: 1522-1528.

46. Le Bastard Q, Al-Gawith GA, Grégoire M, Chapelet G, Javaudin F, Dailly E, et al. Systematic review: Human gut dysbiosis induced by non-antibiotic prescription medications. Aliment Pharmakoi Ther. 2018; 47: 332-345.

47. Bahr SM, Tyler BC, Wooldridge N, Butcher BD, Burns TL, Teesch LM, et al. Use of the secondgeneration antipsychotic, risperidone, and secondary weight gain are associated with an altered gut microbiota in children. Trans Psychiatry. 2015; 5: e652.

48. Wang J, Um P, Dickerman BA, Liu J. Zinc, selenium, and depression: A review of the evidence, potential mechanisms and implications. Nutrients. 2018; 10: 584.

49. Singh A, Trumpff C, Genkinger J, Davis A, Spann M, Werner E, et al. Micronutrient dietary intake in Latina pregnant adolescents and its association with level of depression, stress, and social support. Nutrients. 2017; 9: 1212.

50. Eby GA 3rd, Eby KL. Magnesium for treatment-resistant depression: A review and hypothesis. Med Hypotheses. 2010; 74: 649-660.

51. Popper CW. Single-micronutrient and broad-spectrum micronutrient approaches for treating mood disorders in youth and adults. Child Adolesc Psychiatr Clin N Am. 2014; 23: 591-672.

52. Tsuchimine S, Saito M, Kaneko S, Yasui-Furukori N. Decreased serum levels of polyunsaturated fatty acids and folate, but not brain-derived neurotrophic factor, in childhood and adolescent females with depression. Psychiatry Res. 2015; 225: 187-190.

53. Murakami K, Miyake Y, Sasaki S, Tanaka K, Arakawa M. Dietary folate, riboflavin, vitamin B-6, and vitamin B-12 and depressive symptoms in early adolescence: The Ryukyus child health study. Psychosom Med. 2010; 72: 763-768.

54. Dartois LL, Stutzman DL, Morrow M. L-methylfolate augmentation to antidepressants for adolescents with treatment-resistant depression: A case series. J Child Adolesc Psychopharmacol. 2019; 29: 386-391.

55. Tufan AE, Bilici R, Usta G, Erdoğan A. Mood disorder with mixed, psychotic features due to vitamin b12 deficiency in an adolescent: Case report. Child Adolesc Psychiatry Ment Health 2012; 6: 25.

56. Bell DS. Protean manifestations of vitamin D deficiency, part 2: Deficiency and its association with autoimmune disease, cancer, infection, asthma, dermopathies, insulin resistance, and type 2 diabetes. South Med J. 2011; 104: 335-339.

57. Gracious BL, Finucane TL, Friedman-Campbell M, Messing S, Parkhurst MN. Vitamin D deficiency and psychotic features in mentally ill adolescents: A cross-sectional study. BMC Psychiatry. 2012; 12: 38. 
58. Tolppanen AM, Sayers A, Fraser WD, Lewis G, Zammit S, Lawlor DA. The association of serum 25hydroxyvitamin D3 and D2 with depressive symptoms in childhood-A prospective cohort study. J Child Psychol Psychiatry. 2012; 53: 757-766.

59. Högberg G, Gustafsson SA, Hällström T, Gustafsson T, Klawitter B, Petersson M. Depressed adolescents in a case-series were low in vitamin $D$ and depression was ameliorated by vitamin $D$ supplementation. Acta Paediatr. 2012; 101: 779-783.

60. Marsh WK, Penny JL, Rothschild AJ. Vitamin D supplementation in bipolar depression: A double blind placebo controlled trial. J Psychiatr Res. 2017; 95: 48-53.

61. Jaddou HY, Batieha AM, Khader YS, Kanaan SH, El-Khateeb MS, Ajlouni KM. Depression is associated with low levels of 25-hydroxyvitamin D among Jordanian adults: Results from a national population survey. Eur Arch Psychiatry Clin Neurosci. 2012; 262: 321-327.

62. Kunz M, Severino C, Andreazza AC, Salvador M, Ceresér KM, Gomes FA, et al. Elevated serum superoxide dismutase and thiobarbituric acid reactive substances in different phases of bipolar disorder and in schizophrenia. Prog Neuropsychopharmacol Biol Psychiatry. 2008; 32: 1677-1681.

63. Atkuri K, Mantovani JJ, Herzenberg LA, Herzenberg LA. N-acetylcysteine-A safe antidote for cysteine/glutathione deficiency. Curr Opin Pharmacol. 2007; 7: 355-359

64. Tomko RL, Gilmore AK, Gray KM. The role of depressive symptoms in treatment of adolescent cannabis use disorder with N-Acetylcysteine. Addict Behav. 2018; 85: 26-30.

65. Berk M, Copolov DL, Dean O, Lu K, Jeavons S, Schapkaitz I, et al. N-acetylcyteine for depressive symptoms in bipolar disorder-a double blind randomized placebo-controlled trial. Biol Psychiatry. 2008; 64: 468

66. Berger ME, Smesny S, Kim SW, Davey CG, Rice S, Sarnyai Z, et al. Omega-6 to omega-3 polyunsaturated fatty acid ratio and subsequent mood disorders in young people with at-risk mental states: A 7-year longitudinal study. Transl Psychiatry. 2017; 7: e1220.

67. Wozniak J, Faraone SV, Chan J, Tarko L, Hernandez M, Davis J, et al. A randomized clinical trial of high eicosapentaenoic acid omega-3 fatty acids and inositol as monotherapy and in combination in the treatment of pediatric bipolar spectrum disorders: A pilot study. J Clin Psychiatry. 2015; 76 : 1548-1555.

68. Trebaticka J, Hradečná Z, Böhmer F, Vaváková M, Waczulíková I, Garaiova I, et al. Emulsified omega-3 fatty-acids modulate the symptoms of depressive disorder in children and adolescents: $\mathrm{A}$ pilot study. Child Adolesc Psychiatry Ment Health. 2017; 11: 30.

69. Fristad MA, Young AS, Vesco AT, Nader ES, Healy KZ, Gardner W, et al. A randomized controlled trial of Individual family psychoeducational psychotherapy \& omega-3 fatty acids in youth with subsyndromal bipolar disorder. J Child Adolesc Psychopharmacol. 2015; 25: 764-774.

70. Fristad MA, Vesc AT, Young A, Fristad MA. Pilot RCT of omega-3 and Individual-Family psychoeducational psychotherapy for children and adolescents with depression. J Clin Child Adolesc Psychol. 2017; 45: 1025-1037.

71. Arnold LE, Young A, Belury MA, Cole RM, Gracious B, Seidenfeld AM, et al. Omega-3 fatty acid plasma levels before and after supplementation: Correlations with mood and clinical outcomes in the omega 3 and therapy studies. J Child Adoles Psychopharmacol. 2017; 27: 223-233. 
72. Vesco AT, Young AS, Arnold LE, Fristad MA. Omega-3 supplementation associated with improved parent-rated executive function in youth with mood disorders: Secondary analyses of the omega 3 and therapy (OATS) trials. J Child Psychol Psychiatry. 2018; 59: 628-636.

73. Wallace $C$, Milev R. The effects of probiotics on depressive symptoms in humans: A systematic review. Ann Gen Psychiatry. 2017; 16: 14.

74. Dickerson F, Adamos M, Katsafanas E, Khushalani S, Origoni A, Savage C, et al. Adjunctive probiotic microorganisms to prevent rehospitalization in patients with acute mania: $A$ randomized controlled trial. Bipolar Disord. 2018; 20: 614-621.

75. Messaoudi M, Violl N, Bisso JF, Desor D, Javelot H, Rougeot C. Beneficial psychological effects of a probiotic formulation (Lactobacillus helveticus R0052 and Bifidobacterium longum R0175) in healthy human volunteers. Gut Microbes. 2011; 2: 256-261).

76. Akkasheh G, Kashani-Poor Z, Tajabadi-Ebrahimi M, Jafari P, Akbari H, Taghizadeh M, et al. Clinical and metabolic response to probiotic administration in patients with major depressive disorder: $\mathrm{A}$ randomized, double-blind, placebo-controlled trial. Nutrition. 2016; 32: 315-320.

77. Salazar N, Dewulf EM, Neyrinck AM, Bindels LB, Cani PD, Mahillon J, et al. Inulin-type fructans modulater intestinal Bifodobacterium species populations and decrease short-chain fatty acids in obese women. Clin Nutr. 2015; 34: 493-501.

78. Dahiya DK, Puniya M, Shandilya UK, Dhewa T, Kumar N, Kumar S, et al. Gut microbiota modulation and its relationship with obesity using prebiotic fibers and probiotics: A review. Front Microbiol. 2017; 8: 563

79. Kazemi A, Noorbala AA, Azam K, Eskandari MH, Djafarian K. Effect of probiotic and prebiotic vs placebo on psychological outcomes in patients with major depressive disorder: A randomized clinical trial. Clin Nutr. 2018; 38: 522-528.

80. Amirani E, Milajerdi A, Mirzaei H, Jamilian H, Mansournia MA, Hallajzadeh J, et al. The effects of probiotic supplementation on mental health, biomarkers of inflammation and oxidative stress in patients with psychiatric disorders: A systematic review and meta-analysis of randomized controlled trials. Complement Ther Med. 2020; 49: 102361.

81. Luoto R, Kalliomaki M, Laitinen K, Isolauri E. The impact of peri natal probiotic intervention on the development of overweight and obesity: Follow up from birth to 10 years. Int J Obes. 2010; 34 : 1531-1537

82. Pärtty A, Kalliomäki M, Wacklin P, Salminen S, Isolauri E. A possible link between early probiotic intervention and the risk of neuropsychiatric disorders later in childhood: A randomized trial. Pediatr Res. 2015; 77: 823-828.

83. Ghaderi A, Asbaghi O, Reiner Z, Kolahdooz F, Amirani E, Mirzaei H, et al. The effects of saffron (Crocus sativus L.) on mental health parameters and C-reactive protein: A meta-analysis of randomized clinical trials. Complement Ther Med. 2020; 48: 102250.

84. Panossian AG. Adaptogens in mental health disorders. Psych Clin North Am. 2013; 36: 49-64.

85. Weng S, Tang J, Wang G, Wang X, Wang H. Comparison of the addition of Siberian ginseng (acanthopanax senticosus) versus fluoxetine to lithium for the treatment of bipolar disorder in adolescents: A randomized, double-blind trial. Curr Ther Res Clin Exp. 2007; 68: 280-290.

86. Potter M, Moses A, Wozniak J. Alternative treatments in pediatric bipolar disorder. Child Adolesc 
Psychiatr Clin N Am. 2009; 18: 483-514.

87. Wurglics M, Westerhoff K, Kaunzinger A, Wilke A, Baumeister A, Dressman J, et al. Comparison of German St. John's wort products according to hyperforin and total hypericin content. Am Pharm Assoc (Wash). 2001; 41: 560-566.

88. Findling RL, McNamara NK, O'Riordan MA, Reed MD, Demeter CA, Branicky LA, et al. An open-label pilot study of St. John's wort in juvenile depression. J Am Acad Child Adolesc Psychiatry. 2003; 42: 908-914.

89. Simeon J, Nixon MK, Milin R, Jovanovic R, Walker S. Open-label pilot study of St. John's wort in adolescent depression. J Child Adolesc Psychopharmacol. 2005; 15: 293-301.

90. Schaller JL, Thomas J, Bazzan AJ. SAMe use in children and adolescents (letter). Eur Child Adolesc Psychiatry. 2004; 13: 332-334.

91. Kondo DG, Sung YH, Hellem TL, Delmastro KK, Jeong EK, Kim N, et al. Open label uridine for treatment of depressed adolescents with bipolar. J Child Adolesc Psychopharmacol. 2011; 21: 171175.

92. Kok BE, Coffey KA, Cohn MA, Catalino LI, Vacharkulksemsuk T, Algoe SB, et al. How positive emotions build physical health: Perceived positive communications account for the upward spiral between positive emotions and vagal tone. Psycho Sci. 2013; 24: 1123-1132.

93. Cahn BR, Goodm, Peterson CT, Maturi R, Mills PJ. Yoga, meditation and mind-body health: Increased BDNF, cortisol awakening response, and altered inflammatory marker expression after a 3-month yoga and meditation retreat. Front Hum Neurosci. 2017; 11: 315.

94. Tolahunase MR, Sagar R, Faiqs M, Dada R. Yoga-and meditation-based lifestyle intervention increases neuroplasticity and reduces severity of major depressive disorder: A randomized controlled trial. Restor Neurol Neurosci. 2018; 36: 423-442.

95. Ignácio ZM, Da Silva RS, Plissari ME, Quevedo J, Réus GZ. Physical exercise and neuroinflammation in major depressive disorder. Mol Neurobiol. 2019; 56: 8323-8335.

96. Hughes CW, Barnes S, Barnes C, DeFina LF, Nakonezny P, Emslie GJ. Depressed adolescents treated with exercise (DATE): A pilot randomized controlled trial to test feasibility and establish preliminary effects sizes. Ment Health Phys Act. 2013; 6: 119-131.

97. Merker M, Felder M, Gueissaz L, Bolliger R, Tribolet $P$, Kägi-Braun N, et al. Association of baseline inflammation with effectiveness of nutritional support among patients with disease-related malnutrition-a secondary analysis of a randomized controlled clinical trial. JAMA Netw Open. 2020; 3: e200663.

98. Hanaway P. Gastrointestinal dysfunction, chronic disease and the functional medicine matrix model. Presented at the GI Advanced Practice odules. October 25th, 2018. Nashville, TN. 
OBM Integrative and Complementary Medicine 2020; 5(4), doi:10.21926/obm.icm.2004040

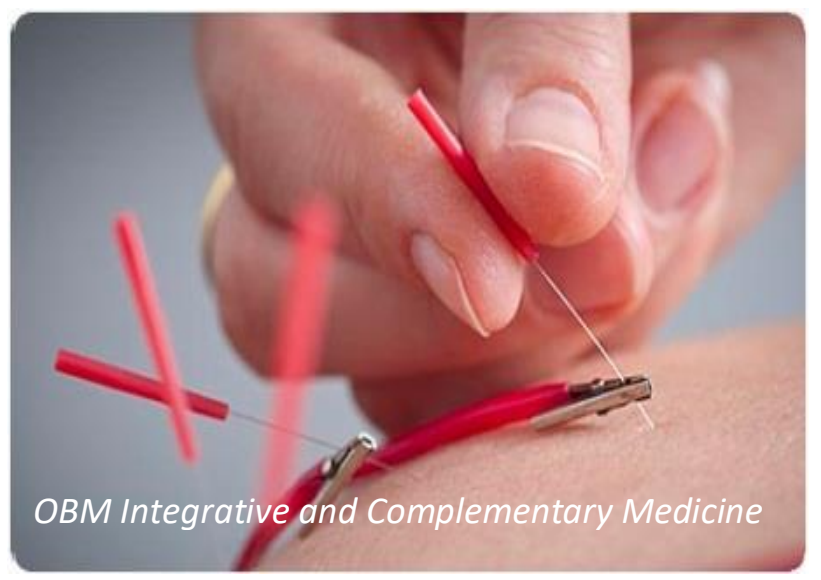

Enjoy $O B M$ Integrative and Complementary Medicine by:

1. Submitting a manuscript

2. Joining in volunteer reviewer bank

3. Joining Editorial Board

4. Guest editing a special issue

For more details, please visit:

http://www.lidsen.com/journals/icm 